\title{
ON VECTOR-VALUED HARDY MARTINGALES AND A GENERALIZED JENSEN'S INEQUALITY
}

\author{
ANNELA R. KELLY and BRIAN P. KELLY
}

Received 24 June 2002

\begin{abstract}
We establish a generalized Jensen's inequality for analytic vector-valued functions on $\mathbb{T}^{N}$ using a monotonicity property of vector-valued Hardy martingales. We then discuss how this result extends to functions on a compact abelian group $G$, which are analytic with respect to an order on the dual group. We also give a generalization of Helson and Lowdenslager's version of Jensen's inequality to certain operator-valued analytic functions.
\end{abstract}

2000 Mathematics Subject Classification: 43A17, 42A45, 60G42.

1. Introduction. We consider generalizations of the classical Jensen's inequality

$$
\int_{0}^{2 \pi} \log \left|f\left(e^{i \theta}\right)\right| \frac{d \theta}{2 \pi} \geq \log \left|\int_{0}^{2 \pi} f\left(e^{i \theta}\right) \frac{d \theta}{2 \pi}\right|
$$

for functions analytic on the closed unit disk of the complex plane.

For the following, let $G$ be a nonzero connected compact abelian group with Haar measure $\lambda$ normalized so that $\lambda(G)=1$. We use $\Gamma$ to denote the discrete dual group. By [11, Theorem 24.25], since $G$ is connected, $\Gamma$ is a torsion-free group. This is necessary and sufficient for the existence of well-defined algebraic orders on $\Gamma$.

The seminal paper of Helson and Lowdenslager [9] introduced a concept of generalized analyticity which has received much attention in the recent literature (see [1, 2, 3, 4, 5]). In [9], they introduced the theory of analytic functions on $G$ where analyticity is defined in terms of functions with Fourier transform supported on a "positive set" $\mathscr{P}$ on the dual group $\Gamma$. Furthermore, [9] contains versions of Jensen's inequality for matrix-valued functions, in particular, Theorems 13 and 14 therein. More precisely, Helson and Lowdenslager obtained the following result.

Proposition 1.1 [9, page 192]. Suppose for some $n$ that $B$ is a function defined on the unit circle of the complex plane that is square summable with 
respect to $\lambda$ and analytic with values in the set of $n \times n$ matrices. Then

$$
\int_{G} \log |\operatorname{det}(B)(x)|^{2} d \lambda \geq \log \left|\operatorname{det}\left(\int_{G} B(x) d \lambda\right)\right|^{2},
$$

where we take $\log (0)=-\infty$.

In [9], this result directly implies a related Jensen's inequality for certain analytic functions with range in a suitable finite-dimensional trace class. As a corollary of our main theorem, we obtain a generalization of this version of Jensens's inequality to much more general spaces of operators.

For our main result, we use martingale theory to prove Jensen's inequality for analytic functions with values in an arbitrary complex Banach space. Hardy martingales were first introduced by Garling in [7]. It has become apparent from $[4,7]$ that Hardy martingales represent a probabilistic counterpart to certain analytic functions on products of tori. In [4], Hardy martingales provided the means to prove a generalized Jensen's inequality for scalar-valued functions. In the present paper, we follow a similar approach by establishing a monotonicity property and then discussing how this implies the desired version of Jensen's inequality.

2. Hardy martingales and Jensen's inequality. We begin by introducing the terminology and notations used in this paper. We denote the set of all complex numbers by $\mathbb{C}$. A set $\mathscr{P} \subset \Gamma$ is an order on the dual group $\Gamma$ whenever it satisfies the following:

(a) $\mathscr{P}+\mathscr{P} \subset \mathscr{P}$,

(b) $\mathscr{P} \cup(-\mathscr{P})=\Gamma$,

(c) $\mathscr{P} \cap(-\mathscr{P})=\{0\}$, where for $B \subset \Gamma,-B=\{-b: b \in B\}$.

As a special case, we use $\mathbb{T}$ to denote the circle group $\left\{e^{i t}: 0 \leq t<2 \pi\right\}$ with normalized Lebesgue measure and identify its dual with $\mathbb{Z}$, the set of integers. Fix $N$ to be a positive integer. We do much of our work with the compact abelian group $\mathbb{T}^{N}$ with normalized Haar measure on $\mathbb{T}^{N}$ denoted by $\mu_{N}$. Similarly as in [7], for functions defined on $\mathbb{T}^{N}$ we often use the reverse lexicographical order on the dual group $\mathbb{Z}^{N}$, that is,

$$
\mathscr{P}_{N}^{*}=\{0\} \cup\left(\bigcup_{j=1}^{N}\left\{\left(n_{1}, \ldots, n_{j}, 0, \ldots, 0\right) \in \mathbb{Z}^{N}: n_{j}>0\right\}\right) .
$$

Let $X$ be a Banach space with norm $\|\cdot\|_{X}$, or simply $\|\cdot\|$. Suppose that $(\Omega, \mathscr{F}, \mu)$ is a general measure space. Whenever $f: \Omega \rightarrow X$ is strongly measurable, $\int_{\Omega} f d \mu$ denotes the Bochner integral. Let $L^{1}(\Omega, X)$ be the Banach space of strongly measurable functions $f: \Omega \rightarrow X$ such that $\|f\|_{1}<\infty$, where $\|f\|_{1}=$ $\int_{\Omega}\|f\|_{X} d \mu<\infty$. When $X=\mathbb{C}$, the field of scalars, we simply write $L^{1}(\Omega)$. 
For $f \in L^{1}(G, X)$, define the Fourier transform $\hat{f}: \Gamma \rightarrow X$ by $\hat{f}(\chi)=$ $\int_{G} f(x) \overline{\chi(x)} d \lambda(x)$. A vector-valued function on $\mathbb{T}$ is analytic if its Fourier transform vanishes on the negative integers. Fundamentals of the theory of analytic vector-valued functions on $\mathbb{T}$ were introduced in [6], and after that several mathematicians have studied the properties of

$$
H^{1}(\mathbb{T}, X)=\left\{f \in L^{1}(\mathbb{T}, X): \hat{f} \text { vanishes for } n<0\right\} .
$$

We generalize the definition above to the functions defined on a compact abelian group $G$, whose dual group is ordered by $\mathscr{P}$. We say $f \in L^{1}(G, X)$ is analytic with respect to $\mathscr{P}$ if the Fourier transform $\hat{f}$ vanishes off $\mathscr{P}$. Define the corresponding Hardy space by

$$
H_{\mathscr{P}}^{1}(G, X)=\left\{f \in L^{1}(G, X): \hat{f} \text { vanishes off } \mathscr{P}\right\} .
$$

Garling introduced vector-valued Hardy martingales in [7] and used them to prove several properties of analytic functions on $\mathbb{T}^{N}$ (with respect to the order $\left.\mathscr{P}_{N}^{*}\right)$. We now recall the relevant definitions and properties for the reader's convenience.

First, let $\mathscr{F}_{0}=\left\{\varnothing, \mathbb{T}^{N}\right\}$, while for $1 \leq j \leq N$, let $\mathscr{F}_{j}$ be the $\sigma$-algebra generated by the first $j$ coordinate functions $\mathscr{F}_{j}=\sigma\left\{e^{i \theta_{1}}, \ldots, e^{i \theta_{j}}\right\}$. Whenever $\mathscr{F}_{F}$ is a sub$\sigma$-algebra of $\mathscr{F}_{N}$, we denote the conditional expectation with respect to $\mathscr{F}$ by $\mathbb{E}(\cdot \mid \mathscr{F})$. A martingale $\left(g_{j}\right)$ on $\mathbb{T}^{N}$ with values in $X$ is called a Hardy martingale if $\mathbb{E}\left(g_{j+1} e^{i n \theta_{j+1} \mid \mathscr{F}_{j}}\right)=0$ for $n>0$ and all $j=0, \ldots, N-1$.

If $f \in L^{1}\left(\mathbb{T}^{N}, X\right)$, define $f_{j}=\mathbb{E}\left(f \mid \mathscr{F}_{j}\right)$ for $j=0,1,2, \ldots, N$. For $j=1, \ldots, N$, the function $f_{j}$ is constructed from $f$ by projecting the Fourier transform of $f$ onto $\mathbb{Z}^{j}$. Here, $\mathbb{Z}^{j}$ is identified with the following subgroup of $\mathbb{Z}^{N}$ :

$$
\mathbb{Z}^{j}=\left\{\left(n_{1}, \ldots, n_{N}\right) \in \mathbb{Z}^{N}: n_{k}=0 \text { for } k=j+1, \ldots, N\right\} .
$$

From the discussion preceding [7, Theorem 1], we can conclude that

$$
H_{\mathscr{P}_{N}^{*}}^{1}\left(\mathbb{T}^{N}, X\right)=\left\{f \in L^{1}\left(\mathbb{T}^{N}, X\right):\left(f_{j}\right) \text { is a Hardy martingale }\right\} .
$$

Let $d_{0}(f)=f_{0}=\int_{\mathbb{T}^{N}} f d \mu_{N}$ while $d_{j}(f)=f_{j}-f_{j-1}$ for $j=1, \ldots, N$. This gives a martingale difference decomposition

$$
f=\sum_{j=0}^{N} d_{j}(f) .
$$

Garling showed in [7] that $f \in H_{\mathscr{P}_{N}^{*}}^{1}\left(\mathbb{T}^{N}, X\right)$ if and only if for $j=1, \ldots, N, d_{j}(f)$ has a formal Fourier series expansion of the form

$$
d_{j}(f)=\sum_{k=1}^{\infty} f_{j, k}\left(\theta_{1} \ldots, \theta_{j-1}\right) e^{i k \theta_{j}},
$$


where $f_{j, k}\left(\theta_{1}, \ldots, \theta_{j-1}\right)$ is a function of $\theta_{1}, \ldots, \theta_{j-1}$. Hence we have that $f \in$ $H_{\mathscr{P}_{N}^{*}}^{1}\left(\mathbb{T}^{N}, X\right)$ if and only if for $j=1,2, \ldots, N$ the function $d_{j}(f)$ belongs to $H^{1}(\mathbb{T}, X)$ when considered as a function of $\theta_{j}$ only.

Proposition 2.1. Suppose that $f \in H_{\mathscr{P}_{N}^{*}}^{1}\left(\mathbb{T}^{N}, X\right)$. Then for $0 \leq n \leq N-1$,

$$
\int_{\mathbb{T}^{N}} \log \left\|\sum_{j=0}^{n} d_{j}(f)\right\| d \mu_{N} \leq \int_{\mathbb{T}^{N}} \log \left\|\sum_{j=0}^{n+1} d_{j}(f)\right\| d \mu_{N} .
$$

Proof. Let $\mathbb{D}=\{z \in \mathbb{C}:|z|<1\}$. If $g: \mathbb{D} \rightarrow X$ is analytic in the sense of being strongly differentiable on $\mathbb{D}$, then from the discussion on [8, page 89], we have, for $0<r<1$,

$$
\log \|g(0)\| \leq \frac{1}{2 \pi} \int_{\mathbb{T}} \log \left\|g\left(r e^{i \theta}\right)\right\| d \theta
$$

As in [6], we can identify each function $g \in H^{1}(\mathbb{T}, X)$ with a Bochner integrable function analytic on $\mathbb{D}$ by defining

$$
g\left(r e^{i \theta}\right)=g * P_{r}(\theta)=\frac{1}{2 \pi} \int_{T} g(t) P_{r}(\theta-t) d t
$$

for $0<r<1$ and $0<\theta<2 \pi$, where $P_{r}(\theta)$ is the Poisson kernel. From the discussion preceding Proposition 2.1 herein, we notice that as a function of $\theta_{n+1}$, the function $\sum_{j=0}^{n+1} d_{j}(f)$ is in $H^{1}(\mathbb{T}, X)$. Hence $\sum_{j=0}^{n+1} d_{j}(f) * P_{r}$ is an analytic function on $\mathbb{D}$, and we can apply (2.9). Next, using [6, Proposition 1], we can take $r=1$ in (2.9) which gives us

$$
\log \left\|\sum_{j=0}^{n+1} d_{j}(f) * P_{0}\right\| \leq\left(\frac{1}{2 \pi} \int_{\theta_{n+1}} \log \left\|\sum_{j=0}^{n+1} d_{j}(f)\right\| d \theta_{n+1}\right) .
$$

Since for $j=0, \ldots, n, d_{j}(f)$ is constant with respect to $\theta_{n+1}$ and $d_{n+1} * P_{0}=$ 0 , we can rewrite inequality (2.11) as

$$
\frac{1}{2 \pi} \int_{\theta_{n+1}} \log \left\|\sum_{j=0}^{n} d_{j}(f)\right\| d \theta_{n+1} \leq\left(\frac{1}{2 \pi} \int_{\theta_{n+1}} \log \left\|\sum_{j=0}^{n+1} d_{j}(f)\right\| d \theta_{n+1}\right) .
$$

The desired conclusion follows immediately from (2.12).

THEOREM 2.2 (Jensen's inequality). Let $G$ be a compact abelian group with an order $\mathscr{P}$ on the dual group $\Gamma$. Given a function $f \in H_{\mathscr{P}}^{1}(G, X)$, we have

$$
\left\|\int_{G} f(x) d \lambda(x)\right\| \leq \exp \left(\int_{G} \log \|f(x)\| d \lambda(x)\right) .
$$


Proof. First, we note that [4, Lemma 3.2] shows that it is sufficient to obtain the result for a function in a dense subspace of $H_{\mathscr{P}}^{1}(G, X)$. Let $f \in H_{\mathscr{P}}^{1}(G, X)$ be an analytic trigonometric polynomial, that is, $f=\sum_{k=1}^{m} x_{k} X_{k}$ where, for $1 \leq k \leq$ $m, x_{k} \in X$ and $\chi_{k} \in \mathscr{P}$. We first discuss reducing the proof to the case when $G=\mathbb{T}^{N}$ for some suitable $N$ and $\mathscr{P}=\mathscr{P}_{N}^{*}$. Applying the Weil formulas (see [10]) as in [4, page 194], we can construct a positive integer $N$, an order $\mathscr{P}(f)$ on $\mathbb{Z}_{N}$, and a trigonometric polynomial $f^{\dagger}=\sum_{k=1}^{m} x_{k} \chi_{k}^{\dagger} \in H_{\mathscr{P}(f)}^{1}\left(\mathbb{T}^{N}, X\right)$ such that

$$
\int_{G} f d \lambda=\int_{\mathbb{U}^{N}} f^{\dagger} d \mu_{N}, \quad \int_{G} \log |f| d \lambda=\int_{\mathbb{U}^{N}} \log \left|f^{\dagger}\right| d \mu_{N} .
$$

Clearly, it suffices to establish the desired result for $f^{\dagger}$. Also as proved in [4], there exists an isomorphism $\phi: \mathbb{T}^{N} \rightarrow \mathbb{T}^{N}$ with adjoint $\phi^{*}: \mathbb{Z}^{N} \rightarrow \mathbb{Z}^{N}$ such that for $j \in\{1, \ldots, m\}, \phi^{*}\left(\chi_{j}\right) \in \mathscr{P}_{N}^{*}$. Then we have that $f^{\dagger} \circ \phi \in H_{\mathscr{P} *(N)}^{1}\left(\mathbb{T}^{N}, X\right)$. At this point, we can see that (2.13) will hold for $f$ if and only if the corresponding statement holds for $f^{\dagger} \circ \phi$.

All that remains is to prove (2.13) for the case $f \in H_{\mathscr{P}_{N}^{*}}^{1}\left(\mathbb{T}^{N}, X\right)$. But this follows directly from repeated application of Proposition 2.1 since $d_{0}=\int_{\mathbb{T}^{N}} f d \mu_{N}$.

In [9], Helson and Lowdenslager obtained versions of Jensen's inequality for matrix-valued functions. With our main theorem we can now extend their results as stated below.

COROLLARY 2.3. Let $\mathscr{H}$ be any seperable Hilbert space and let $\Phi_{1}$ be the space of trace-class operators on $\mathcal{H}$. Then, if $f: G \rightarrow I_{1}$ is analytic on $G$, we have

$$
\int_{G} \log (\operatorname{tr}|f(x)|) d \lambda \geq \log \left(\operatorname{tr}\left|\int_{G} f(x) d \lambda\right|\right) .
$$

Proof. We only need to note that the space of trace-class operators $\Phi_{1}$ acting on a separable Hilbert space $\mathscr{H}$ is a Banach space under the norm $\|A\|_{1}=$ $\operatorname{tr}|A|$ where $|A|$ denotes the absolute value of an operator (see [12, Sections VI.4, VI.6]).

REMARK 2.4. We find it rather interesting that we do not need to place geometric assumptions on the Banach space such as Burkholder's UMD condition. In papers such as $[3,5]$, the UMD condition arises because we seek what amounts to certain bounded projections from $L^{1}(G, X)$ to $H_{\mathscr{P}}^{1}(G, X)$. This requires more concern for the geometry of the Banach space $X$ and the related vector-valued function spaces. Our work deals strictly with properties of the functions in $H_{\mathscr{P}}^{1}(G, X)$, which is closer in spirit to the work in [6].

Also note that in our case, analyticity is defined in terms of the Fourier transform. This leads to several aspects of the proofs being readily adapted from work previously done for scalar-valued functions. 
ACKNOwLEDGment. The authors would like to thank the referee for their comments which proved very helpful in delineating the full scope of our results.

\section{REFERENCES}

[1] N. Asmar, E. Berkson, and T. A. Gillespie, Representations of groups with ordered duals and generalized analyticity, J. Funct. Anal. 90 (1990), no. 1, 206-235.

[2] N. Asmar and E. Hewitt, Marcel Riesz's theorem on conjugate Fourier series and its descendants, Proceedings of the Analysis Conference, (Singapore, 1986) (S. T. L. Choy et al., eds.), North-Holland Math. Stud., vol. 150, NorthHolland, Amsterdam, 1988, pp. 1-56.

[3] N. Asmar, B. P. Kelly, and S. Montgomery-Smith, A note on UMD spaces and transference in vector-valued function spaces, Proc. Edinburgh Math. Soc. (2) 39 (1996), no. 3, 485-490.

[4] N. Asmar and S. Montgomery-Smith, Hardy martingales and Jensen's inequality, Bull. Austral. Math. Soc. 55 (1997), no. 2, 185-195.

[5] E. Berkson, T. A. Gillespie, and P. S. Muhly, Generalized analyticity in UMD spaces, Ark. Mat. 27 (1989), no. 1, 1-14.

[6] A. V. Bukhvalov and A. A. Danilevich, Boundary properties of analytic and harmonic functions with values in a Banach space, Mat. Zametki 31 (1982), no. 2, 203-214 (Russian), English translation Mat. Notes 31 (1982), 104110.

[7] D. J. H. Garling, On martingales with values in a complex Banach space, Math. Proc. Cambridge Philos. Soc. 104 (1988), no. 2, 399-406.

[8] M. Heins, Hardy Classes on Riemann Surfaces, Lecture Notes in Mathematics, no. 98, Springer-Verlag, Berlin, 1969.

[9] H. Helson and D. Lowdenslager, Prediction theory and Fourier series in several variables, Acta Math. 99 (1958), 165-202.

[10] E. Hewitt and K. A. Ross, Abstract Harmonic Analysis. Vol. II: Structure and Analysis for Compact Groups. Analysis on Locally Compact Abelian Groups, Die Grundlehren der Mathematischen Wissenschaften, vol. 152, SpringerVerlag, New York, 1970.

[11]_ Abstract Harmonic Analysis. Vol. I, Grundlehren der Mathematischen Wissenschaften, vol. 115, Springer-Verlag, Berlin, 1979.

[12] M. Reed and B. Simon, Methods of Modern Mathematical Physics. I. Functional Analysis I, Academic Press, New York, 1980.

Annela R. Kelly: Department of Mathematics and Physics, University of Louisiana at Monroe, Monroe, LA 71209, USA

E-mail address: ake11y@u 1m.edu

Brian P. Kelly: Department of Mathematics and Physics, University of Louisiana at Monroe, Monroe, LA 71209, USA

E-mail address: ke11y@u 1m. edu 


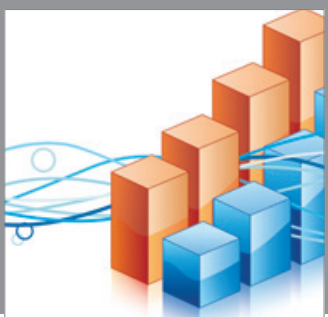

Advances in

Operations Research

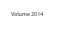

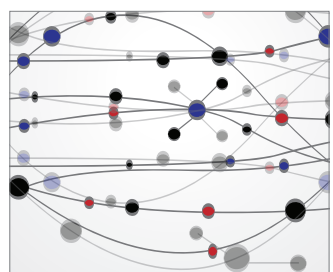

\section{The Scientific} World Journal
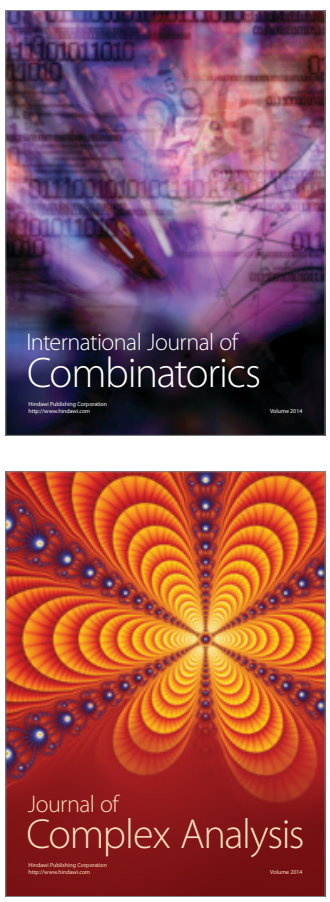

International Journal of

Mathematics and

Mathematical

Sciences
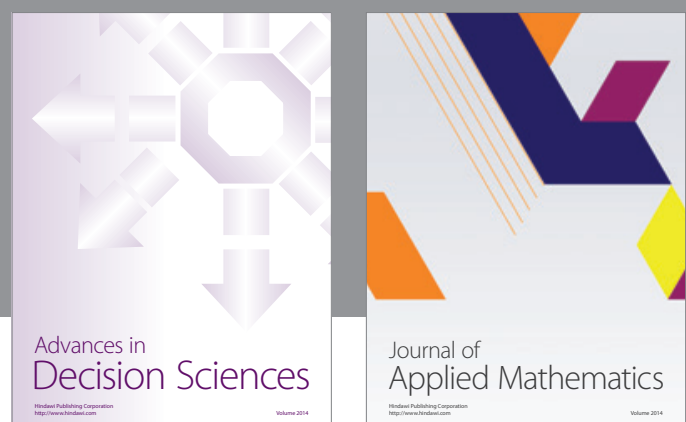

Journal of

Applied Mathematics
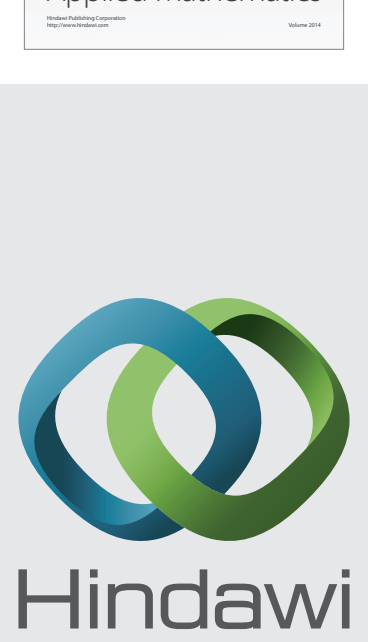

Submit your manuscripts at http://www.hindawi.com
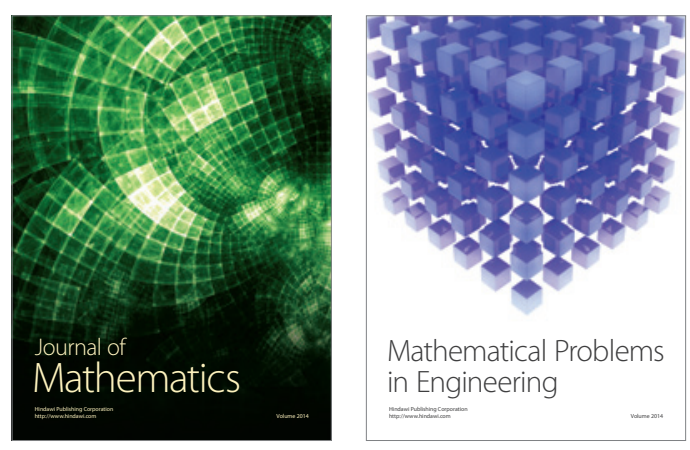

Mathematical Problems in Engineering
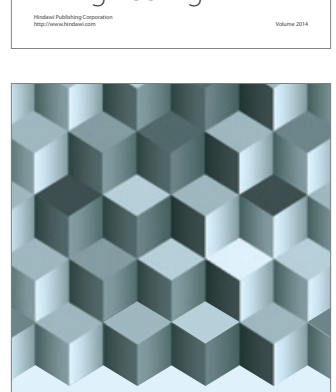

Journal of

Function Spaces
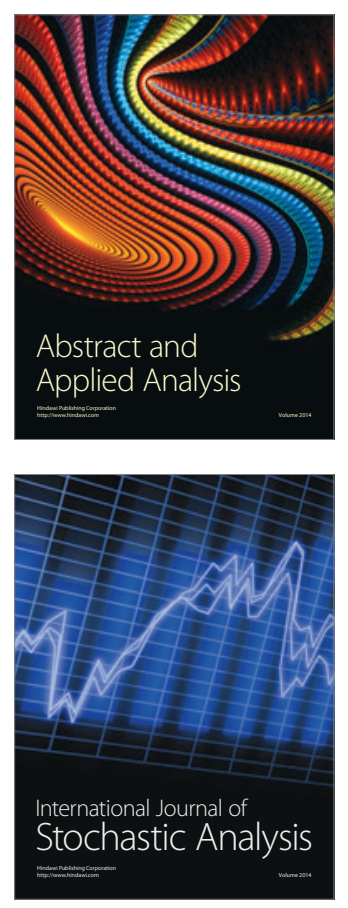

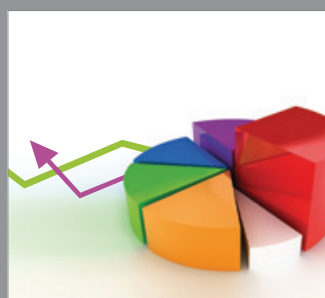

ournal of

Probability and Statistics

Promensencen
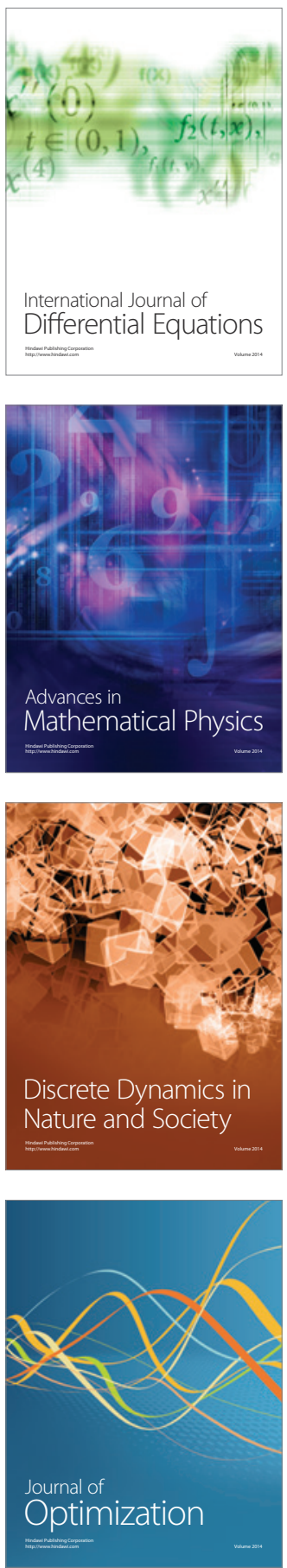